\title{
TEORÍA DEL CASO Y SINTAXIS MINIMISTA ${ }^{1}$
}

\author{
Ángel J. GAllego \\ Universitat Autònoma de Barcelona
}

\section{RESUMEN}

Este trabajo discute el papel que desempeña el Caso en la teoría lingüística, ofreciendo diferentes formas de reflejar tal fenómeno desde la perspectiva de la gramática generativa. Por tanto, se revisan mecanismos como la Rección, la concordancia SPEC-núcleo o la Atracción. El artículo dedica especial atención tanto al sistema de Sonda-Meta de Chomsky 2000, 2001, como a la idea de Pesetsky y Torrego 2001 de que lo que llamamos Caso es en realidad un rasgo de tiempo/aspecto que aparece «de manera equivocada» en entornos nominales, considerando su relevancia para la llamada «intervención defectiva» y los «efectos de extracción» en lenguas como el inglés, el islandés o el español.

Palabras clave: Caso estructural, Caso inherente, Sonda, Meta, Acuerdo, intervención defectiva, Emparejamiento, Valoración, tiempo, isla, defectividad.

\section{Abstract}

This paper discusses the role of Case in linguistic theory, offering the different ways of implementing such a phenomenon from the perspective of generative grammar. Accordingly, mechanisms such as Government, SPEC-head agreement, or Attract are reviewed. The article focuses on both Chomsky's 2000, 2001 Probe-Goal framework and Pesetsky and Torrego's 2001 hypothesis that Case is actually a tense/aspect feature «misplaced» in nominal environments, considering their relevance for socalled «defective intervention» and «CED effects» in languages like English, Icelandic, or Spanish.

Key Words: Structural Case, Inherent Case, Probe, Goal, Agree, defective intervention, Match, Valuation, tense, island, defectivity.

\section{INTRODUCGIÓN}

Un aspecto central en la investigación de orientación generativista (desde Chomsky 1955/1975 hasta la actualidad) es la «Teoría del Caso», el módulo de la gramática encargado de regular la asignación a los Sin-

\footnotetext{
${ }^{1}$ Agradezco a M. Lluïsa Hernanz, M. Carme Picallo y Esther Torrego sus comentarios a una versión previa de este trabajo. Me gustaría dar las gracias especialmente a José M. Brucart y Juan Uriagereka, no solo por sus sugerencias y observaciones sobre las cuestiones que aquí discuto, sino también por su constante apoyo. La redacción de este trabajo se ha beneficiado de las ayudas del MEC-FEDER (HUM2006-13295-C02-02) y la Generalitat de Catalunya (2005SGR-00753).
} 
tagmas Nominales/Determinante (sDs, de aquí en adelante) de información morfológica relacionada con las funciones gramaticales (sujeto, objeto, etc.). La noción de «Caso», cuyo papel dentro de la teoría sintáctica generativista puede describirse como menor hasta el advenimiento del marco teórico de «Principios y Parámetros» (PyP; cf. Chomsky 1981, 1986a, 1986b), ha demostrado ser crucial para el estudio de dos de las propiedades sintácticas más idiosincrásicas de las lenguas naturales: la localidad y el fenómeno del desplazamiento ${ }^{2}$.

Siguiendo la tradición inaugurada por el modelo de PyP, la investigación generativista más reciente ha reforzado la idea de que la morfología casual no solo se relaciona con la propiedad de dislocación de los objetos sintácticos, sino que también permite delimitar dominios locales de computación (los «ciclos» o «fases»). La formalización precisa de la Teoría del Caso ha sufrido constantes modificaciones a lo largo de sus poco más de veinticinco años de historia, pero en general hay un alto grado de consenso sobre su importancia. En las siguientes páginas pretendo ofrecer un resumen de las diferentes formulaciones de la Teoría del Caso, desde el modelo de «Rección y Ligamiento» (RL; cf. Chomsky 1981, 1986a, 1986b y Chomsky y Lasnik 1995) hasta el «Programa Minimista» (PM; cf. Chomsky 1995, 2000, 2001, 2004, 2008), mencionando, para finalizar, la reciente hipótesis de Pesetsky y Torrego 2001 de que lo que llamamos «Caso» es en realidad un rasgo de tiempo/aspecto que poseen los determinantes.

El trabajo se organiza de la siguiente manera: el segundo apartado está destinado a revisar las diferentes estrategias propuestas para asignar Caso estructural a lo largo del modelo PyP, discutiendo nociones como «Rección», «Sonda», «Meta» o «Atracción», y explicando en cada caso qué motivó el cambio de análisis; pondré especial énfasis en la formulación más reciente de la «Teoría del Caso» (i. e., el sistema de SondaMeta presentado en Chomsky 2000, 2001), aprovechando para estudiar detenidamente los llamados «efectos de intervención». En el tercer apartado resumo los aspectos básicos de la propuesta de Pesetsky y Torrego 2001, su interesante relación con el llamado Caso inherente y sus implicaciones para las teorías de localidad. El cuarto apartado recoge, por último, las principales conclusiones del artículo.

\footnotetext{
${ }^{2}$ La noción de «Caso» en el marco de la sintaxis generativista, de hecho, fue ignorada hasta el envío de una carta, escrita por Jean Roger Vergnaud a Noam Chomsky (17 abril de 1977), tras la publicación del artículo «Filters and Control» en la revista Linguistic Inquiry. En tal artículo, Chomsky y Howard Lasnik proponían una serie de restricciones sobre posibles secuencias de símbolos. Así, por ejemplo, se argüía que una estructura como (i) debía ser considerada (mediante estipulación) como agramatical, como indica el asterisco:

(i) * SN V (INF) SN

En su carta, Vergnaud le hacía notar a Chomsky que restricciones como la de (i) (y otras similares) se podían derivar teniendo en cuenta la noción tradicional de Caso.
} 


\section{Teorías del Caso: De «Rección y Ligamiento» al Minimismo}

En Chomsky 1981, p. 49 se formula el «Filtro de Caso», una restricción encargada de regular la distribución de los SDs manifestados fonéticamente:

(1) Filtro de Caso?:

*sD si SD tiene contenido fonético y no tiene Caso

Chomsky 1981 asume que la existencia del Caso es independiente de su manifestación fonética, por lo que se puede expresar de manera abstracta. En palabras de Chomsky y Lasnik 1995, pp. 110-111:

En algunas lenguas (sánscrito, latín, ruso, etc.), el Caso se manifiesta morfológicamente, pero en otras tiene escasa (inglés, francés, etc.) o nula (chino, etc.) realización explícita. En línea con nuestra formulación general, asumimos que el Caso siempre está presente de manera abstracta. En lenguas de Caso nominativo / acusativo, el sujeto de una oración flexionada recibe Caso «nominativo»; el objeto de un verbo transitivo recibe Caso «acusativo» [...]; y el objeto de una pre/posposición recibe Caso «oblicuo».

En un principio, la Teoría del Caso fue diseñada para explicar la agramaticalidad de ejemplos como los de (2), donde la presencia explícita de sDs en entornos verbales no flexionados ocasionaba agramaticalidad:

(2) a. *It seems [Susan to be happy]

b. $*$ I am proud [Bill to be here]

Nótese que, a diferencia de los ejemplos de (2), los de (3) -que únicamente añaden propiedades flexivas en el verbo- sí legitiman un sujeto explícito.

(3) a. It seems [that Susan is happy]

b. I am proud [that Bill is here]

De la mera comparación de (2) y (3) se puede concluir que los infinitivos bloquean por lo general la aparición de un SD explícito en posición de sujeto. El fenómeno descrito es uniforme en los llamados infinitivos de «Control» (4a; que recurren a un formativo nulo, PRO) ${ }^{4}$, pero no en los de «Elevación» (4b), ni en los de «Marcaje Excepcional de Caso» (MEC) (4c):

(4) a. John tried [ ${ }_{\mathrm{SC}}$ PRO to call Mary] Control

\footnotetext{
${ }^{3}$ En (1) sustituyo el rótulo «SN» (Sintagma Nominal) por «SD» (Sintagma Determinante); el cambio es meramente terminológico, sin repercusiones para la discusión que sigue.

${ }^{4}$ No entro en detalles sobre PRo, la categoría vacía que aparece en los infinitivos de Control. Hasta Chomsky y Lasnik 1995 se suponía que PRo era un elemento que no recibía Caso, pero desde ese trabajo se asume que recibe una variedad especial de Caso que estos autores denominan «Caso nulo». Para discusión sobre estas cuestiones, cf. Hornstein 2003.
} 
b. John ${ }_{\mathrm{i}}$ seemed $\left[_{\mathrm{ST}} \mathrm{t}_{\mathrm{i}}\right.$ to like Mary] Elevación

c. John wants $\left[_{\mathrm{ST}}\right.$ Mary to call him] Marcaje Excepcional de Caso

La particularidad de (4b) y (4c) radica en la transparencia sintáctica de la cláusula subordinada: en ambos casos, un elemento funcional de la matriz puede asignar Caso al sujeto de la subordinada («nominativo» en casos de elevación, «acusativo» en casos de MEC) $)^{5}$.

\subsection{Dos tipos de Caso}

En su primera formulación, la Teoría del Caso se centraba en los llamados «casos estructurales», nominativo y acusativo (o ergativo y absolutivo para las lenguas ergativas, como el vasco); el resto de Casos se subsumían bajo el rótulo de «oblicuo». Más tarde, Chomsky 1986b introduce una distinción crucial: la que diferencia el «Caso estructural» del «Caso inherente» ${ }^{6}$. Tal distinción, entre otras cosas, pretendía explicar la siguiente asimetría:

(5) a. [Destruyeron [la ciudad]]

b. [Destrucción [*(de) la ciudad]]

La observación tradicional en relación a (5) es fácil de expresar: los verbos asignan Caso; los sustantivos no. ¿Qué hace diferente a las categorías v y N en cuanto al Caso? Probablemente sea el rasgo [ $\pm \mathrm{N}]$, propuesto en Chomsky 1981 para caracterizar las categorías v, N, A y P (las tres primeras, léxicas; la última, funcional), el responsable de la asimetría de (5), aunque es difícil ver cómo puede reformularse esta idea en términos actuales.

Pero la principal novedad de Chomsky 1986b, p. 193 fue la propuesta de que todas las categorías sintácticas eran asignadores potenciales de Caso: v y $\mathrm{T}$ (en la formulación del momento, Flex) asignarían Caso estructural, mientras que N, A y $\mathrm{P}$ asignarían Caso inherente. Crucialmen-

\footnotetext{
${ }^{5}$ En los casos de elevación, el proceso es bastante obvio, puesto que el sujeto literalmente se desplaza al especificador del nudo T matriz. ¿Qué sucede en los infinitivos de MEc? En (4c) he supuesto que el SD Mary permanece in situ, recibiendo acusativo «a larga distancia»; véase, no obstante, Lasnik 1999 para un análisis en el que Mary asciende a la posición de objeto de la oración matriz, en el espíritu del análisis de «elevación a objeto» de Postal 1974.

${ }^{6}$ En los trabajos del marco teórico minimista la noción de Caso inherente ha sido explorada de manera marginal; de hecho, no conozco ni una propuesta firme sobre esta variedad de Caso, algo que seguramente se debe a la desaparición del nivel de representación donde se codificaba la «Estructura Profunda» (cf. Chomsky 1993). Una suerte análoga ha corrido la noción de «Caso partitivo", propuesta en Belletti 1988 para ejemplos como (i), donde un SD indefinido concuerda con un verbo inacusativo:

(i) Llegan niños.

El motivo es, en gran medida, el mismo: el Caso partitivo se asignaba en la Estructura Profunda. Para una discusión, cf. Boeckx 2000 y las referencias allí citadas.

Existe una división ulterior del Caso inherente en «Caso idiosincrásico» $\mathrm{y}$ "Caso semántico» (cf. Svenonius 2002).
} 
te, Chomsky 1986b define el Caso inherente en relación a la «Teoría Temática»: es decir, para Chomsky 1986b, un SD recibe Caso inherente del predicado que lo selecciona (y que le asigna papel temático). El Caso estructural, por el contrario, puede ser asignado por elementos que no seleccionan semánticamente a $\operatorname{los} \operatorname{sDs}^{7}$. El par de (6) sirve para ilustrar este aspecto.

(6) a. María tiene [ [a su hijo] [en el hospital] ]

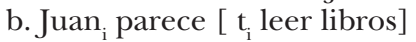

En (6), los SDs a su hijo y Juan reciben Caso acusativo y nominativo respectivamente. Lo importante de estos ejemplos es que los asignadores de Caso son el verbo (o $v^{*}$, como veremos más adelante) y $\mathrm{T}$, pero los predicados de estos SDs son otros: a su hijo es seleccionado por en el hospital, y Juan por leer. El panorama es distinto en (7), donde los predicados del SD Juan son los mismos que le asignan Caso (mediante la preposición $d e$ ): el $\mathrm{N}$ cansancio y el A cansado.

(7) a. [El [cansancio [de Juan]]]

b. [Cansado [de Juan]]

Es importante subrayar otro aspecto de (7): desde la perspectiva de Chomsky 1986b, son N y A los que asignan Caso a Juan, aunque lo hagan mediante la preposición (semánticamente inerte) de. Plausiblemente (aunque Chomsky 1986b no entra en esta discusión), los ejemplos de (8) deberían ser tratados aparte:

(8) a. María llamó [después de la cena]

b. María jugó [con Elena]

Bajo el supuesto de que llamary jugar no seleccionan argumento interno (o lo incorporan, en el sentido de Hale y Keyser 2002), debemos concluir que, en estos ejemplos, son después y con los que asignan tanto Caso como papel temático. Tal conclusión es razonable en más de un sentido, pero sobre todo permite reflejar que estas preposiciones (a diferencia de $d e$ ), tienen una semántica independiente ${ }^{8}$. La distinción tiene consecuencias interesantes en términos de localidad: si, como

\footnotetext{
${ }^{7}$ Otra relación significativa entre la Teoría del Caso y la Teoría Temática era la «Condición de Visibilidad», adoptada por Chomsky 1986b, p. 94 siguiendo sugerencias de Joseph Aoun.

${ }^{8}$ Mi exposición simplifica mucho los datos. En (i) y (ii), por ejemplo, son los sustantivos viaje y denuncia los que asignan Caso al término de la preposición, por mucho que esta tenga, en un sentido intuitivo, más semántica que la de que veíamos en (7):

i) [El [viaje [a Washington D.C.]]]

ii) $[\mathrm{La}[$ denuncia [contra tu empresa]]]

Obviamente, digo esto sin aportar una caracterización más refinada de qué quiere decir, en el caso de las preposiciones, tener una semántica independiente. La cuestión, en el fondo, se reduce a averiguar qué (tipo de) semántica tienen las preposiciones. Dejando a un lado las preposiciones que son meras marcas de Caso (la $a$ estudiada en Torrego 1998, por ejemplo), la bibliografia reciente (cf. Hale 1986, Hale y Keyser 2002, y Demirdache y Uribe-Etxebarria 2000) suele diferenciar dos valores para las preposiciones: el de «coincidencia central» (en, debajo, detrás, etc.) y el de «coincidencia terminal» (por, desde, hacia, etc.).
} 
defendemos en Gallego y Uriagereka 2007, solo los sDs (o scs) que reciben Caso inherente son islas ${ }^{9}$, los datos de (9) se siguen de manera natural $^{10}$.

(9)a. $\left[{ }_{\mathrm{SC}}\right.$ De qué escritor ${ }_{\mathrm{i}}$ has leído $\left[_{\mathrm{SD}}\right.$ muchas novelas $\left.\mathrm{t}_{\mathrm{i}}\right]$ ]?

b. ${ }^{*}\left[{ }_{\mathrm{SC}}\right.$ De qué amigos has comprado regalos $\left[_{\mathrm{SP}}\right.$ para los hijos $\left.\left.\mathrm{t}_{\mathrm{i}}\right]\right]$ ?

\subsection{Caso: ¿mecanismos o configuraciones?}

Aún no he mencionado cuáles son los mecanismos encargados de asignar Caso. En una primera etapa del modelo RL, se trabajaba con la hipótesis de que el Caso estructural era asignado mediante un mecanismo denominado «Rección» (Government), cuya definición adapto de Chomsky y Lasnik 1995, p. $79^{11}$ :

(10) Rección:

$\alpha$ rige a $\beta$ si $\alpha$ manda-c a $\beta$ y no hay una categoría $\gamma$ tal que esta se interponga entre $\alpha$ y $\beta$.

Dada la definición de (10), se suponía que el verbo ejercía Rección sobre el objeto, asignándole acusativo (11a), y la misma lógica se aplicaba en los casos de MEc (11b), siempre y cuando el nudo ST no se interpusiese entre v y el SD sujeto de la oración subordinada:

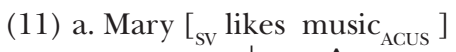

b. John believed [ ${ }_{\mathrm{ST}}^{\uparrow}$ Mary $_{\mathrm{ACUS}}$ to like music ]

En cuanto al nominativo, este se asignaba mediante el ascenso del sujeto al especificador de $\mathrm{T}$ (Flex), donde se creaba una configuración de especificador-núcleo. En términos técnicos, se puede afirmar que el sujeto también recibía Caso bajo rección, pero para ello era necesario recurrir a una variedad más laxa de mando-c que se extendiese a los especificadores: el «mando-m». Tal relación fue ampliamente explotada en Chomsky 1993, donde se suponía que tanto nominativo como acusativo se asignaban de manera simétrica, en configuraciones de especificador-núcleo; en particular, se suponía que dos proyecciones de

\footnotetext{
${ }^{9}$ Una isla es un dominio que no permite dependencias sensibles al contexto (concordancia, transformaciones de movimiento, etc.) entre un elemento interno y otro externo. Cf. Boeckx 2003 para un resumen pormenorizado de estas cuestiones.

${ }^{10}$ Modificando ideas de Boeckx 2003 y Chomsky 2000, 2001, en Gallego y Uriagereka 2007 suponemos que solo puede haber (sub-)extracción de un dominio sintáctico si este puede establecer concordancia con los núcleos funcionales $\mathrm{C}$ y $v^{*}$.

${ }^{11}$ Este mecanismo pretendía reformular la idea tradicional de que el verbo rige sus dependientes (i. e., los elementos con los que se relaciona). A lo largo del modelo RL, la definición de Rección se vio sometida a revisiones cada vez más complejas (cf. Uriagereka 1988), debido a la presencia de esta noción en el resto de módulos o subteorías de la gramática.
} 
concordancia $\left(\right.$ Conc $_{\mathrm{S}}$ y Conc $_{\mathrm{O}}$ ) alojarían a sujeto y a objeto, asignándoles $\mathrm{Caso}^{12}$ :

(12)

$$
\begin{aligned}
& \text { SConc }_{\mathrm{S}} \\
& \mathrm{SD}_{\text {NOM }} \text { Conc }_{\mathrm{S}} \text {, } \\
& \text { Conc }_{\mathrm{S}} \text { ST } \\
& \mathrm{SD}_{\text {ACus }} \text { Conc }_{\mathrm{O}} \text {, } \\
& \text { Conc }_{\mathrm{S}} \quad \mathrm{SV}
\end{aligned}
$$

Este análisis presenta aspectos interesantes -y otros problemáticos-. Lo crucial es que (12) es una propuesta virtualmente idéntica a las de una serie de investigadores (encabezados por Koizumi 1995 y Lasnik 1999), quienes, recuperando observaciones de Postal, argüían que, al igual que los sujetos, los objetos del inglés se desplazan para recibir Caso.

Chomsky 1995, la primera reformulación del Programa Minimista, modifica el análisis de (12) esgrimiendo problemas conceptuales con las proyecciones de concordancia. Según Chomsky 1995, este tipo de proyecciones no pueden existir como tales, puesto que no eran interpretables en la interfaz semántica (lo que Chomsky llama «rasgos- $\varphi$ » no se interpreta en los verbos, sino en los nombres). Esto debe entenderse bien: Chomsky no niega la existencia de esta información, lo que niega es que pueda constituir una proyección sintáctica independiente.

En ese mismo trabajo (1995, p. 262), Chomsky propone que, si la asignación de Caso debe entenderse como un proceso de comprobación de rasgos, solo estos deben ser manipulados por la sintaxis; así pues, no deberían moverse sDS, sino sus rasgos:

La idea intuitiva de fondo es que la operación de Movimiento está regida por consideraciones morfológicas: el requerimiento de que algún rasgo $\mathrm{R}$ debe cotejarse. La operación mínima, entonces, debería mover tan solo el rasgo R: deberíamos restringir $\alpha$ de la operación Muévase $\alpha$ a rasgos léxicos.

Suponiendo que el movimento se desencadena «desde arriba», mediante algún núcleo funcional, Chomsky 1995 reformula la operación de asignación de Caso en términos de «Atracción» (Attract): para asignar nominativo, por ejemplo, se asumía que $\mathrm{T}$ atraía un rasgo nominal del SD sujeto. Una predicción inmediata de esta concepción era que el Caso podía asignarse «a larga distancia», sin movimiento.

\footnotetext{
${ }^{12}$ Este análisis tenía su origen en propuestas de Emonds 1978 y Pollock 1989, posteriormente modificadas por Adriana Belletti y el propio Chomsky (cf. Belletti 1990 y Chomsky 1991), de que el nudo molecular Flexión debía escindirse en dos proyecciones independientes: Tiempo y Concordancia. En un primer momento, solo había un nudo de Concordancia (concordancia de sujeto); en Chomsky 1991, la idea de asignar Caso mediante una proyección de Concordancia se extiende al objeto, lo cual permitía reflejar de manera natural las observaciones que había hecho Richard Kayne (cf. Kayne 1989) a propósito de la concordancia con participios. Sin duda, el trabajo de Pollock 1989 es el germen del llamado «Proyecto Cartográfico» de Rizzi 1997.
} 
No obstante, esto no es factualmente cierto, como se ve en el caso del inglés; Chomsky 1995 se da cuenta de ello y sugiere que el movimiento estricto -lo que él llama «arrastre» (pied-piping) - es una operación parasitaria, motivada por imposiciones de la interfaz morfo-fonológica. Así, para explicar por qué el SD sujeto debe ascender al especificador de $\mathrm{T}$, Chomsky aduce que el desplazamiento debe concebirse como una «estrategia de reparación morfológica»: en términos metafóricos, el rasgo atraído por T deja al SD «incompleto», por lo que este tiene que subir a una posición cercana a la del núcleo que le ha «arrancado» su rasgo (el especificador de $\mathrm{T}$, en este caso) para poder así reintegrarlo en su estructura morfológica. El proceso sería más o menos como describo en (13).

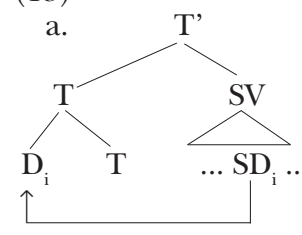

Atracción de un rasgo D

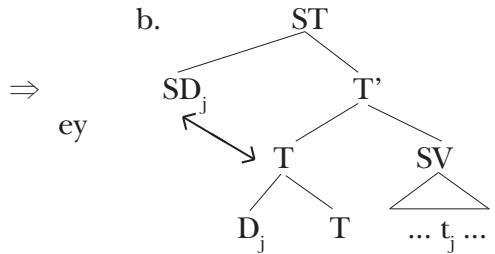

Reparación Morfológica del SD

Nótese que bajo esta perspectiva, el movimiento sería un proceso destinado a evitar problemas en la interfaz morfo-fonológica, algo que era coherente con la concepción (por entonces) de que el lenguaje tenía dos imperfecciones: la morfología y el desplazamiento (cf. Chomsky 2000). Según (13), explicar el movimiento como un fenómeno morfológico permitía relacionar las dos supuestas imperfecciones y reducirlas a una sola.

Podemos dejar la discusión aquí. En este apartado he intentado repasar las diversas estrategias para asignar Caso en el modelo RL y en las primeras formulaciones del PM. Como hemos visto, el Caso se concebía como el resultado de relacionar los SDs con un elemento de tipo funcional (las proyecciones de concordancia, esencialmente) mediante una operación específica. En RL este papel era desempeñado por la franquicia teórica de aquel modelo: la Rección. Con el minimismo, la Rección se elimina como operación independiente (la causa era obvia: los mismos resultados podían obtenerse mediante conceptos de la «Teoría de la X-barra», que eran necesarios de manera independiente a la Teoría del Caso), y el Caso pasa a concebirse como el reflejo de una configuración. Finalmente, Chomsky 1995 retoma la idea de que sea una operación (y no una configuración) la que asigne Caso, y propone el mecanismo de Atracción, pero se encuentra, de nuevo, con el problema del EPP ${ }^{13}$ : el (ex-

\footnotetext{
${ }^{13}$ El origen de esta etiqueta se remonta a lo que en Chomsky 1981 se denomina «Principio $\mathrm{P}$ » y servía para referirse a la necesidad de lenguas como el inglés de tener la posición preverbal de sujeto ocupada de manera explícita. En Chomsky 1982, el «Principio P» pasó a llamarse
} 
traño) requerimiento de que el SPEC-T sea ocupado de manera explícita en lenguas como el inglés.

\subsection{Caso y concordancia}

Pese a sus ventajas conceptuales, Chomsky pronto se dio cuenta de los problemas técnicos que planteaba la operación de Atracción. Especialmente preocupante era el hecho de que el desplazamiento de rasgos se concibiese como un proceso de movimiento de núcleos (esto es, el movimiento de rasgos era como el movimiento del verbo a $\mathrm{T}$, digamos), lo cual violaba la «Condición de Extensión» de Chomsky 1993: la restricción de que toda operación sintáctica tenga algún efecto sobre la estructura, extendiéndola.

En Chomsky 2000 se reformula Atracción, eliminando el desplazamiento literal de rasgos por una operación abstracta denominada «Acuerdo» (Agree). Simplificando ligeramente la cuestión, Chomsky asume que los núcleos $\mathrm{T}$ y $v^{*}$ (una categoría funcional que encabeza los Svs transitivos, llamada «verbo ligero») se introducen en la sintaxis con rasgos nominales: los rasgos- $\varphi$. Chomsky 2000 razona que estos rasgos no son interpretables en $\mathrm{T}$ y $v^{*}$, por lo que entran en la computación sin valor (algo que se marcaría de la siguiente manera: [_R]). La necesidad de obtener un valor antes de transferir la estructura al componente semántico haría -arguye Chomsky- que $\mathrm{T}$ y $v^{*}$ actuasen como una «Sonda» (Probe) que busca una «Meta» $(\text { Goal })^{14}$.

Chomsky 2000, 2001 impone algunas condiciones sobre el Acuerdo:

(14) a. Sonda y Meta deben estar activos para que Acuerdo actúe.

b. Acuerdo se divide en Emparejamiento y Valoración.

c. Una Sonda debe ser $\varphi$-completa para poder asignar Caso.

Hay varios puntos de (14) que merecen aclaración. En primer lugar, vayamos con la noción de «actividad». Chomsky 2000 supone que la morfología no interpretable hace que los elementos que la manifiestan estén activos, pudiendo participar en operaciones sintácticas; en el caso de los sDs, el Caso estructural es la información que desempeña este

\footnotetext{
«Principio de Proyección Extendido» (Extended Projection Principle o EPP), pues describía un requerimiento que sobrepasaba la formulación del «Principio de Proyección» de Chomsky 1981, al exigir la inserción de elementos que no recibían papel temático, como los expletivos ingl. there o fr. il. Actualmente, el EPP se concibe como un rasgo que permite crear o bien especificadores (cf. Chomsky 2000) o bien especificadores y complementos (cf. Chomsky 2008).

${ }^{14}$ Este punto es importante, porque con él Chomsky está defendiendo la idea de que la interpretabilidad no es importante dentro de la computación sintáctica. En otras palabras, la sintaxis no se preocupa de que un rasgo sea interpretable o no (eso no lo ve la sintaxis, lo ve el componente semántico), sino de algo mucho más formal: de que un rasgo tenga un valor.

Cf. Uriagereka 1998, donde se presenta la idea (metafórica) de que los rasgos no interpretables son «virus» que deben eliminarse del sistema computacional.
} 
papel. Una vez han satisfecho esa necesidad, Chomsky 2000 asume que son desactivados (de hecho, Chomsky es más gráfico, y dice que al recibir Caso, los SD quedan «congelados»): la desactivación hace que no puedan volver a moverse a otra posición (quedan, por decirlo así, inutilizados para propósitos sintácticos). Esa es la explicación que Chomsky 2000 da para ejemplos como (15a), conocidos como «Hiperelevación» (Hyper-raising):

(15) a. *Los niños parecen [ que ti están cansados]

b. Los niños ${ }_{i}$ parecen [ $\mathrm{t}_{\mathrm{i}}$ estar cansados]

En (15), el SD los niños se desplaza desde su posición de sujeto de la oración subordinada hasta la posición de sujeto de la oración matriz. Debe destacarse que el proceso sólo es lícito en (15b), porque sólo en ese caso María no recibe nominativo en la oración subordinada.

La siguiente noción que quiero tratar es la noción de «Emparejamiento» (Match). Chomsky 2000 propone que el proceso de Acuerdo comprende un primer paso en el que hay un encaje de rasgos: un proceso de Emparejamiento, literalmente. Tal y como se define, Emparejamiento es una operación sensible solamente al tipo de rasgo, independientemente de su valor. Para ser específico, los $\operatorname{rasgos}-\varphi$ de $\mathrm{T}$ y $v^{*}$ pueden emparejarse con los sDs sujeto y objeto, como se indica en (16a):

a. $\left[_{\mathrm{SC}} \mathrm{C}\left[{ }_{\mathrm{ST}} \mathrm{T}[\underset{\uparrow}{\varphi}]\left[\mathrm{S}_{v^{*}} \mathrm{SD}[5 \varphi] \quad v^{*}\left[\underset{\uparrow}{\varphi}\left[\left[_{\mathrm{SV}} \mathrm{V} \mathrm{SD}[6 \varphi]\right]\right]\right]\right]\right.\right.$ Emparejamiento

Tras el proceso de Emparejamiento de rasgos- $\varphi$, las Metas transfieren su valor a las Sondas mediante un proceso de «Valoración» ${ }^{15}$ :

b. $\left[_{\mathrm{SC}} \mathrm{C}\left[_{\mathrm{ST}} \mathrm{T}[5 \varphi]\left[\left[_{\mathrm{S}^{*}} \mathrm{SD}[5 \varphi]_{\mathrm{NOM}} v^{*}[6 \varphi]\left[_{\mathrm{SV}} \mathrm{V} \mathrm{SD}[6 \varphi]_{\mathrm{ACUS}}\right]\right]\right]\right]\right.$ Valoración

Es importante subrayar que la Valoración va unida a la asignación de Caso estructural: al encontrar Metas «activas», las Sondas pueden valorar sus rasgos- $\varphi$ y asignar Caso (nominativo al SD sujeto, acusativo al SD objeto). En este sentido, Chomsky 2000 apunta que el sistema de Sonda-Meta reinterpreta la concepción tradicional sobre la concordancia entre sujeto y verbo: es el segundo el que concuerda con el primero, no viceversa.

Antes de abordar cuestiones de localidad, me gustaría destacar dos aspectos de este análisis: uno, la asignación de Caso no invoca ningún tipo de configuración (simplemente mando-c entre Sonda y Meta); y dos, el Caso, como tal, no participa del proceso de Sonda-Meta. Esta última afirmación, que es opuesta a lo que se había asumido hasta ese momento, es explícita en Chomsky 2001, p. 6:

\footnotetext{
${ }^{15}$ Indico en (16) el valor de los rasgos- $\varphi$ mediante un número arbitrario. Un análisis más refinado requeriría separar el haz de rasgos- $\varphi$ en [número] y [persona].
} 
El Caso estructural no es un rasgo de las Sondas $\left(\mathrm{T}, \mathrm{v}^{*}\right)$, sino que se manifiesta con un valor determinado bajo concordancia, siendo después eliminado de la sintaxis. El valor asignado depende de la Sonda: Nominativo de T, Acusativo de $\mathrm{v}^{*}$ (o Ergativo-Absolutivo, con condiciones diferentes). El caso en sí mismo no se somete a Emparejamiento, sino que se elimina tras el Emparejamiento de los rasgos- $\varphi$.

En otras palabras, Chomsky nos invita a suponer que el Caso es un efecto colateral de la concordancia, sin estatus independiente.

Para finalizar esta sección me gustaría centrarme en la propiedad de que un elemento sea « $\varphi$-completo». En el contexto de su sistema de Sonda-Meta del Caso, Chomsky 2000, 2001 argumenta que algunas Sondas no pueden asignar Caso estructural; serían Sondas «defectivas» en relación a sus rasgos nominales, teniendo únicamente [persona] o [número]. Concretamente, Chomsky 2001 establece la siguiente correlación, que de momento es críptica:

(17) a. C selecciona a un T $\varphi$-completo

b. V selecciona a un $\mathrm{T} \varphi$-defectivo

Como puede verse, Chomsky 2001 hace una serie de suposiciones tácitas en (17), principalmente relacionadas con lo que él llama «fases». ¿Qué son las fases? En su intento de simplificar la relación entre el componente léxico y la sintaxis, Chomsky propone que la computación trabaje con subconjuntos de piezas léxicas de manera aislada: las fases, o dominios sintácticos que imponen límites de memoria computacional. Así, Chomsky 2000, 2001, basándose en evidencia de tipo interpretativo (efectos de reconstrucción) y morfológico (proporcionada por los sistemas de Caso y concordancia), asume que solo SC y S $v^{*}$ son fases ${ }^{16}$.

Con esto en mente, volvamos a (17). La suposición más evidente que allí se hace se refiere a las construcciones de MEC y Elevación: en ellas, v selecciona a un T que sería defectivo, debido a la ausencia de sc. Según Chomsky 2001, T puede asignar nominativo solo si está seleccionado por c, algo que no sucede en las estructuras de MEC y Elevación.

Ya hemos hablado de la relación entre c y т. ¿Qué pasa con la que se establece entre $v^{*}$ y V? A diferencia de trabajos anteriores (cf. Chomsky 1995, 2000), Chomsky 2001 propone que todo sv está introducido por un verbo ligero: $v^{*}$ en el caso de las estructuras transitivas, $\mathrm{v}$ en el de las inacusativas ${ }^{17}$. ¿En qué se diferencian $v^{*}$ y $v$ ? Chomsky 2001 supone que solo $v^{*}$ tiene una estructura argumental plena, seleccionando un argu-

\footnotetext{
${ }^{16}$ Existe una relación evidente entre las «fases» de Chomsky 2000, 2001, los «nudos cíclicos» de Chomsky 1973, y las «barreras» de Chomsky 1986a. En todos los casos, hablamos de dominios sintácticos que intentan capturar la idea de "computación composicional cíclica». Cf. Gallego 2007 para discusión.

${ }^{17}$ Esto difiere de manera no trivial de la propuesta de Chomsky 1995, 2000, donde solo las estructuras transitivas tenían $v^{*}$, siendo las inacusativas un sv simple. Chomsky asume que siempre hay un verbo ligero debido a observaciones de Julie Legate (cf. Legate 2003), quien demuestra que también hay efectos de reconstrucción en los sintagmas verbales inacusativos.
} 
mento externo (un Agente o un Experimentante) y asignando Caso acusativo al argumento interno. Chomsky 2001 propone que la distinción debe establecerse en términos de «defectividad»: $v^{*}$ es « $\varphi$-completo", mientras que $v$ es « $\varphi$-defectivo». En este momento es importante recordar la condición (14c), que repito aquí como (18):

(18) Una Sonda debe ser « $\varphi$-completa» para poder asignar Caso.

Para evaluar las consecuencias de (18), veamos cómo se asigna Caso en una estructura inacusativa, por ejemplo. Considérese el ejemplo de (19):

(19) Ha llegado María.

En (19), el SD María se genera como argumento interno del verbo inacusativo llegar. En un primer estadio derivacional, los rasgos- $\varphi$ de v actúan como Sonda, emparejándose con los de María:

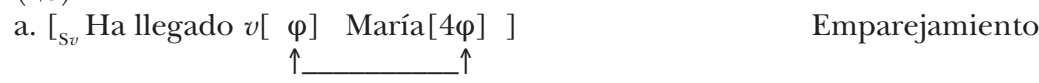

Chomsky 2001 propone que los rasgos de María valoren los de $v$, el cual, no obstante, no puede asignar acusativo, debido a su defectividad.

(20)

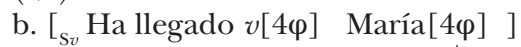

Valoración

La consecuencia es clara: el SD María sigue «activo», pues no ha recibido Caso. La derivación, no obstante, sigue, y la siguiente Sonda que aparece es T, cuyos rasgos- $\varphi$ ejercen, de nuevo, como Sonda. Los rasgos$\varphi$ de т y María se emparejan: los primeros se valoran, y esta vez María recibe Caso.

(21)

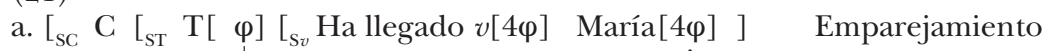

b. $\left[_{\mathrm{SC}} \mathrm{C}\left[_{\mathrm{ST}} \mathrm{T}[4 \varphi]\left[_{\mathrm{S} v}\right.\right.\right.$ Ha llegado $v[4 \varphi]$ María $\left.[4 \varphi]_{\mathrm{NOM}}\right] \quad$ Valoración

Es importante hacer notar que la relación sintáctica de (21) entre $\mathrm{T}$ y el SD María hace un bypass sobre $v$. El hecho no es menor si nos tomamos en serio la idea de que las dependencias sintácticas son estrictamente locales (para que a y c se relacionen no puede interponerse ningún otro elemento). Esta idea ya estaba presente en el modelo RL, y fue profusamente estudiada en Rizzi 1990. Intuitivamente, podemos reflejar esta hipótesis de la siguiente manera:

(22) $A_{[R]} \ldots B_{[R]} \quad \cdots C_{[R]}$

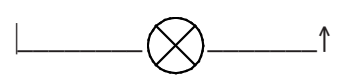


(22) quiere transmitir la idea de que A y c no pueden «comunicarse sintácticamente» si se interpone un в que hable el mismo idioma (donde «hablar el mismo idioma» significa «tener los mismos rasgos»).

En (21) surge este tipo de problema, al menos potencialmente. La pregunta relevante es: ¿puede bloquear $v$ la relación entre т y María? En principio sí, porque $v$ cumple los requisitos para contar como Meta para $\mathrm{T}$ : tiene un conjunto de rasgos- $\varphi$ valorado (defectivo, sí, pero valorado). El problema está en que no hay bloqueo, y la oración es buena ${ }^{18}$, contrariamente a lo que esperaríamos.

Pero el problema es más general, como le hicieron notar a Chomsky John Frampton, Sam Gutmann, Charles Yang y Julie Legate (cf. Gutmann y otros 2000). En concreto, el mismo problema de intervención se da en ejemplos como (23), donde asumiré que los participios tienen rasgos- $\varphi$ (excepto [persona], debido a su naturaleza adjetival) y rasgos de Caso (visibles en lenguas como el latín, pero no el español o el inglés):

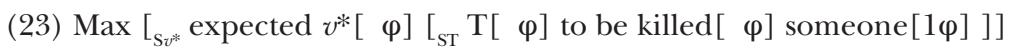

El ejemplo de (23) es ligeramente diferente y más complejo que el que se nos planteaba en (21). En (23) Gutmann y otros 2000 suponen que el participio killed hace de Sonda y se empareja con someone: tras este primer Emparejamiento, killed valora sus rasgos- $\varphi$, pero, al igual que someone, no recibe Caso:

(24) $\operatorname{Max}\left[_{S_{\vartheta^{*}}}\right.$ expected $v^{*}[\varphi]\left[_{\mathrm{ST}} \mathrm{T}[\varphi]\right.$ to be killed $[1 \varphi]$ someone $\left.\left.[1 \varphi]\right]\right]$

La siguiente Sonda a tener en cuenta es el nudo T subordinado, que es defectivo. T se empareja con someone, y sucede lo mismo que antes: la Sonda valora sus rasgos- $\varphi$ y, además, fusiona a someone como su especificador (debido al EPP obligatorio del inglés). El escenario, en este punto, es el siguiente:

(25) Max $\left[_{S^{*}}\right.$ expected $v^{*}[\varphi]\left[_{S \mathrm{~T}}\right.$ someone $_{\mathrm{i}}[1 \varphi] \mathrm{T}[1 \varphi]$ to be $\left.\left.\operatorname{killed}[1 \varphi] \mathrm{t}_{\mathrm{i}}\right]\right]$

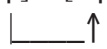

Lo que necesitamos ahora es que $v^{*}$, la siguiente Sonda, no solo se empareje con someone (para asignarle acusativo), sino también con el participio killed, que se encuentra «enterrado» en la estructura. Es en este punto donde surge un problema como el de (21), pero más grave: someone (que, a diferencia de $v$, es $\varphi$-completo) se interpone entre $v^{*} \mathrm{y}$

\footnotetext{
${ }^{18}$ Como he dicho, $v$ no tiene todo el paquete de rasgos nominales (es defectivo), lo cual podría tener algo que decir en la ausencia de bloqueo. Chomsky 2000, p. 149 observa un caso similar en el cual el expletivo there (que, para Chomsky, solo tiene un rasgo de [persona] no valorado) no genera bloqueo sintáctico.

(i) There look as though there are $\{$ three men / *a man $\}$ in the room.

Lo importante de (i) es que en la oración matriz $\mathrm{T}$ y there deben obtener un valor del SD three men (como refleja la concordancia en plural), pero para ello deben hacer un bypass sobre el there intermedio.
} 
killed. De nuevo, la oración debería ser inaceptable, debido a un efecto de intervención.

¿Hay alguna explicación alternativa? Trabajos recientes han intentado resolver esta complicación técnica apelando a un proceso de «Acuerdo Múltiple» (Multiple Agree) que se establecería entre una Sonda y más de una Meta, de manera simultánea (cf. Hiraiwa 2005). El proceso se puede describir como lo hago en $(26)^{19}$ :

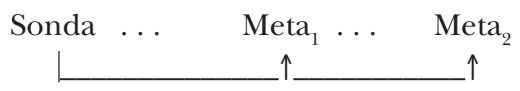

Hiraiwa 2005 observa que un análisis en términos de Acuerdo Múltiple es empíricamente correcto y conceptualmente superior a los anteriores (en los que se recurría a la noción de «equidistancia»; cf. nota 19). El único escenario en el que el Acuerdo entre la Sonda de (26) y la segunda Meta es imposible es aquel en que la primera Meta ha sido valorada por una Sonda ( $\varphi$-completa) diferente. Es decir, la configuración de (27) no permitiría un proceso de Acuerdo Múltiple, según Hiraiwa 2005:

(27) Sonda $_{1} \ldots$ Sonda $_{2} \ldots$ Meta $_{1} \ldots$ Meta $_{2}$

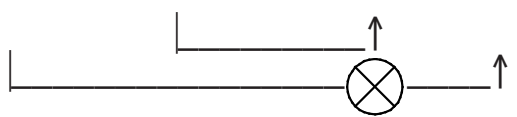

En (27), al haber sido desactivada por la Sonda ${ }_{2}$, la Meta interfiere

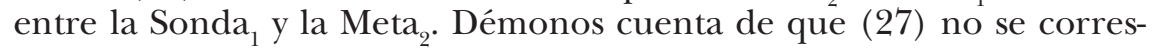
ponde con ninguno de los dos escenarios que hemos observado hasta el momento, que repito ahora. En el primero, el posible interventor es $v$; en el segundo, someone:

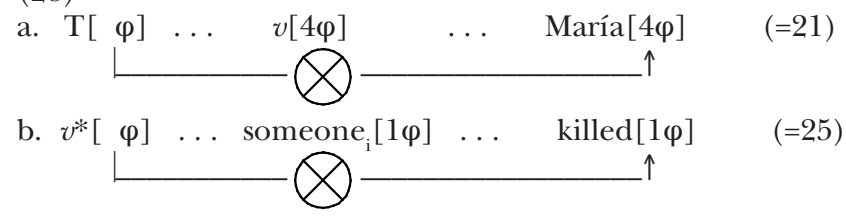

\footnotetext{
19 Quiero recordar aquí que la configuración de (26) no era un problema en Chomsky 1993, 1995, 2000: la Sonda podía hacer un bypass sobre la Meta siempre y cuando las dos Metas formasen parte de un mismo «dominio» (i. e., siempre y cuando las dos Metas fuesen sendos especificadores de un mismo núcleo o especificadores de núcleos diferentes pero combinados mediante movimiento). En los trabajos que he mencionado, Chomsky asumía que esos especificadores eran equidistantes, por lo cual ninguna Meta estaba más cerca de la Sonda que otra. La noción de equidistancia, eliminada en Chomsky 2001, es sin duda interesante, porque era la única manera de explicar que $\mathrm{T}$ asignase nominativo en una configuración en la que el objeto había ascendido por encima del sujeto (el proceso de «Object Shift» de las lenguas escandinavas).
} 
Ya que en ningún caso el posible interventor ha entrado en una relación completa de Acuerdo con otra Sonda, el escenario descrito en (27) es irrelevante. Hay, empero, una diferencia crucial entre los dos interventores de (28): los rasgos- $\varphi$ de v, aunque valorados, no solo son defectivos (v únicamente tendría [persona]), sino que deben eliminarse. Es decir, los rasgos- $\varphi$ de v tienen que ser eliminados de la derivación, pero no así los de someone. Para incorporar esta distinción, formularé (29):

(29) Condición de Intervención:

Solo los rasgos de un SD $\varphi$-completo generan efectos de intervención.

(29) hace que (28a) no sea un problema, a la vez que nos permite analizar (28b) como un ejemplo de Acuerdo Múltiple entre $v^{*}$, someone y killed (los dos últimos recibirían Caso acusativo de $v^{*}$, como deseamos).

Hemos visto, en suma, que es posible evitar los efectos de intervención si se dan las condiciones necesarias. Podríamos preguntarnos, por supuesto, si los efectos de intervención que predice la teoría de Chomsky 2000, 2001 en efecto se dan. El contraste de (30), originalmente observado por Esther Torrego (cf. Torrego 1996), demuestra que sí:

(30) a. Elena ${ }_{\mathrm{i}}$ parece $\left[_{\mathrm{ST}} \mathrm{t}_{\mathrm{i}}\right.$ trabajar mucho]

b. *Elena ${ }_{i}$ me parece $\left[_{S T} t_{i}\right.$ trabajar mucho $]$

En (30) tenemos dos ejemplos con el verbo de elevación parecer, que, de acuerdo con el análisis estándar, trato como un verbo inacusativo que selecciona una estructura oracional defectiva: un sT. Como veíamos más arriba, Chomsky supone que el $\mathrm{T}$ de este tipo de cláusulas no puede asignar nominativo (porque es $\varphi$-defectivo), con lo que el SD Elena establece Acuerdo con el T matriz, desplazándose a su posición de especificador. El proceso no plantea ningún problema en (30a), pero sí en (30b), que, como hace notar Torrego 1996, es agramatical. Suponiendo que parecer puede seleccionar un experimentador (representado por el clítico me en 30b). Torrego 1996 explica el problema de (30b) en términos de intervención: el clítico recibe Caso inherente (como demuestra la contrapartida con el pronombre tónico: me - $a m \hat{\imath}$ ), pero sus rasgos- $\varphi$ son interpretables, por lo que pueden bloquear el proceso de Acuerdo entre el T matriz y Elena, como se ve en (31):

(31) $\mathrm{T}[\varphi] \quad \ldots \quad \operatorname{me}[7 \varphi] \ldots \quad$ Elena $[3 \varphi]$

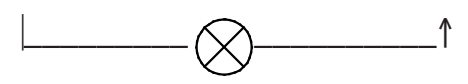

Sorprendentemente, la versión inglesa de (30b) no presenta efectos de intervención: el experimentante (to John) no impide que la T matriz y Mary se relacionen.

(32) Mary $_{\mathrm{i}}$ seems to John $\left[_{\mathrm{ST}} \mathrm{t}_{\mathrm{i}}\right.$ to be gorgeous]

Boeckx 1999 aduce una explicación interesante de la ausencia de 
intervención por parte de to John. Según Boeckx 1999, la preposición to protege al SD JOHN, lo cual permite un bypass limpio.

El análisis de Boeckx 1999 puede mantenerse incluso en ejemplos con los llamados «sujetos caprichosos» (quirky subjects). La bibliografía suele caracterizar a este tipo de sDs como portadores, simultáneamente, de Caso inherente y Caso estructural: el segundo permite que sean sDs activos, pudiendo así desplazarse; el primero evita que concuerden, pero, crucialmente, no que den lugar a efectos de intervención:

(33) Mér \{fannst $/ *$ fundust $\}$ henni leiđast beir. (Islandés) A mí ${ }_{\text {DAT }}\left\{\right.$ parecía / parecían\} ella DAT $_{\text {a }}$ aburrían ellos NOM 'Me parecía que ellos la aburrían (a ella)'

Es decir, en (33) el SD henni impide que el $\mathrm{T}$ matriz se asocie con el sujeto de la oración subordinada: el SD Peir.

El español también posee sujetos caprichosos (cf. Masullo 1992 para discusión), pero salvo en algunos casos particulares estos no desencadenan efectos de intervención. Compárense ambos tipos de sujetos caprichosos: en (34a) a Ana no provoca intervención, pero sí en (34b):

(34) a. Yo sé que a Ana le gustamos nosotros.

b. *A Ana nos le olvidamos nosotros.

Rivero 2004, pp. 495s.

Este apartado ha sido largo. En él he presentado (y discutido con cierta meticulosidad) la última reformulación de la Teoría del Caso en el PM, prestando especial atención a los efectos de intervención que el sistema de Sonda-Meta predice ${ }^{20}$.

No ha habido cambios sustanciales en los trabajos más recientes de Chomsky hasta donde se me alcanza. Cabe destacar, eso sí, una modificación motivada por el empeño de Chomsky por reforzar el estatuto de las fases. Desarrollando una idea esbozada en Chomsky 2004, Chomsky 2008 defiende que los rasgos- $\varphi$ se generan en los núcleos de fase, c y $v^{*}$, desde donde se descargan -mediante un proceso de «herencia»- a $\mathrm{T}$ y $\mathrm{v}$ respectivamente.

Chomsky 2008 da este paso no solo para reforzar el papel de c y $v^{*}$ como loci de todos los rasgos formales, sino porque se ve virtualmente obligado a ello: la única manera de que esos rasgos se eliminen de la derivación es que en el momento de transferencia a los componentes interpretativos, estos pertenezcan al «complemento» de los núcleos fases, ya que solo ese dominio se transfiere; los especificadores, que pertene-

\footnotetext{
${ }^{20}$ No he dicho nada sobre si los efectos de intervención se dan con otro tipo de rasgos. Tradicionalmente, el dato de (i) y (ii) se toma como indicio de que sí:

(i) Who t said what?

(ii) *What did who say t?

Ejemplos como (a) y (b) ilustran la llamada «Condición de Superioridad» (cf. Boeckx 2003).
} 
cen a lo que Chomsky llama «Arista» (Edge), no pueden ser transferidos, pues de lo contrario el movimiento cíclico no sería posible (contrariamente a lo que sabemos). Lo que obliga a Chomsky 2001 a asumir el mecanismo de herencia es, por un lado, la idea de que el borrado de rasgos no interpretables forma parte de los procesos de transferencia y, por el otro, su «Condición de Impenetrabilidad de Fase»:

(35) Condición de Impenetrabilidad de Fase:

El dominio de $\mathrm{X}$ [un núcleo de fase] no es accesible a operaciones en $\mathrm{SY}$; solo X y su arista son accesibles a tales operaciones.

Chomsky 2001, p. 14

(35) es una estipulación en el sistema, pero inevitable si asumimos la hipótesis de que la sintaxis transfiere información a los componentes semántico y fonológico, una idea común desde la «Teoría Estándar» (cf. Chomsky y Lasnik 1977).

La cuestión es cuándo (y cuántas veces) opera ese proceso de transferencia de información. Chomsky 2001 supone que el proceso es estrictamente cíclico, operando «fase a fase»: al final de cada fase, se envía un pedazo de derivación, aquella que corresponde al «complemento» del núcleo de fase; la Arista permanecería en la derivación. Si nos concentramos en el $\mathrm{S} v^{*}$, pongamos por caso, la transferencia «despacharía» el sv, dejando al argumento externo (más otros sintagmas que se hayan desplazado como especificadores externos de $\left.v^{*}\right)$ accesible para las operaciones que tengan lugar en la siguiente fase.

(36)

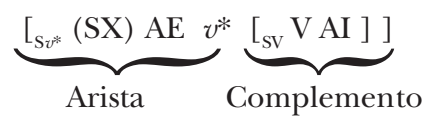

Consecuentemente, debido a (35), el proceso de herencia tiene que ocurrir. Si no fuese así, los rasgos- $\varphi$ de c y $v^{*}$, que son no interpretables, recibirían un valor, pero no se eliminarían del sistema: permanecerían en $\mathrm{c}$ y $v^{*}$, siendo indistinguibles de los interpretables en la siguiente fase.

\section{El Caso como Tiempo/Aspecto}

El apartado anterior ha sido dedicado a lo que podríamos denominar concepciones chomskyanas del Caso (desde Chomsky 1981 hasta la actualidad). Me parece especialmente significativo recordar un aspecto de la última formulación (la de Chomsky 2000, 2001): el Caso, como tal, no existe, siendo un mero reflejo de la concordancia. Hay motivos, empero, para dudar que esto sea así. El primero de ellos tiene que ver 
con el tipo de información que representan Caso por un lado y concordancia por otro: la última indica valores para las propiedades de género, número y persona, pero el Caso no indica nada de eso; el Caso se asocia con otros tres valores: nominativo, acusativo y oblicuo (donde oblicuo subsume una larga lista de Casos no estructurales). De hecho, lo que podríamos preguntarnos es qué tipo de información transmite el Caso. La pregunta es relevante si nos centramos en el Caso estructural, puesto que, a diferencia del Caso inherente, está desprovisto de semántica: recibir nominativo o acusativo no se asocia a interpretación alguna, es una información puramente formal ${ }^{21}$. Hay, además, una segunda pregunta que creo relevante: ¿por qué Chomsky no supone que hay rasgos de Caso independientes de los rasgos- $\varphi$ ? La respuesta resulta clara si se tienen en cuenta los detalles de su sistema de Sonda-Meta: asumir un rasgo de Caso en los sDs le obligaría a asumir la existencia de una contrapartida interpretable en las categorías funcionales, un rasgo de Caso en $\mathrm{T}$ y $v^{*}$ que fuese introducido en la derivación con un valor, lo cual, como acabamos de decir, no tiene sentido: el Caso estructural es el único rasgo formal que siempre es no interpretable.

En una serie de trabajos recientes, Pesetsky y Torrego 2001, 2004, 2007 hacen una propuesta de análisis del Caso en términos de SondaMeta. En concreto, Pesetsky y Torrego 2001 suponen que lo que llamamos Caso es en realidad un rasgo de tiempo:

(37) La Naturaleza del Caso:

El Caso es un rasgo no interpretable $\mathrm{T}$ en $\mathrm{D}^{22}$.

Pesetsky y Torrego 2001, p. 361

Según estos autores, (37) no es algo radical: estamos acostumbrados a la idea de que rasgos puramente nominales (i. e., los rasgos- $\varphi$ ) aparezcan en el verbo; (37) simplemente nos invita a suponer que la imagen especular también es cierta, y que hay rasgos temporales (abstractos) en D. La propuesta es interesante en el sentido de que defiende que todos los rasgos formales tienen una contrapartida potencialmente interpretable: «concordancia» es el nombre que damos a las propiedades de D (es decir, a las propiedades interpretables en D) cuando aparecen en $\mathrm{T}$, mientras que Caso es el que damos a las propiedades de $\mathrm{T}$ (es decir, a las propiedades interpretables en $\mathrm{T}$ ) cuando aparecen en D. Esta concepción, insisto, no es posible en el sistema de Chomsky, donde el Caso no es un rasgo, sino un efecto residual de la concordancia, tal y como explican Pesetsky y Torrego 2007, p 16:

\footnotetext{
${ }^{21}$ Nótese que tampoco serviría asociar la noción de nominativo a la de «sujeto», porque el término «sujeto» describe una relación formal entre un SD y el verbo, no un aspecto nocional (es decir, ser sujeto no puede asociarse a tener tal o cual semántica).

${ }^{22}$ Pesetsky y Torrego 2001 defienden que también C tiene un rasgo [T] no interpretable. De momento dejo esto a un lado, pero será relevante dentro de poco.
} 
El sistema de Chomsky 2000, 2001 no ve el Caso estructural como la versión no interpretable de otro rasgo interpretable. En lugar de eso, es un rasgo sui generis con una relación especial con los rasgos- $\varphi$ : recibe un valor como resultado de la concordancia de rasgos- $\varphi$. Así pues, cuando los rasgos- $\varphi$ de $T$ hacen de Sonda, bajo esta perspectiva, y encuentran una Meta adecuada -por ejemplo, un SD con un conjunto de rasgos- $\varphi$ completo- el rasgo no valorado de Caso del SD recibe un valor, como si fuera una especie de «propina».

Suponiendo que nominativo y acusativo son manifestaciones de rasgos-T, Pesetsky y Torrego 2004 proponen la existencia de una segunda $\mathrm{T}$, seleccionada por $v^{*}$ : una especie de proyección de aspecto por debajo del verbo ligero, como indico en (38). La configuración de (38), curiosamente, no se diferencia mucho de la de Chomsky 1993: solo habría que cambiar las dos proyecciones de tiempo por las dos de concordancia $^{23}$.

(38) $\left[_{\mathrm{SC}} \mathrm{C}\left[_{\mathrm{ST}} \mathrm{T}_{\mathrm{SUJ}}\left[\left[_{\mathrm{S}^{*}} \mathrm{AE} v^{*}\left[_{\mathrm{ST}} \mathrm{T}_{\mathrm{OBJ}}\left[\left[_{\mathrm{SV}} \mathrm{V}\right.\right.\right.\right.\right.\right.\right.$ AI $\left.\left.\left.]\right]\right]\right]$

Pesetsky y Torrego 2004 extienden el análisis del Caso como un rasgo $\mathrm{T}$ a las preposiciones, asumiendo que $\mathrm{P}$ y $\mathrm{T}$ son variantes de la misma megacategoría. Si P contiene un rasgo $\mathrm{T}$ interpretable, el contraste de (39) recibe una explicación inmediata: los objetos reciben Caso del verbo directamente, no de una preposición (en el ejemplo debe descartarse una interpretación partitiva de la preposición $d e$, que es imposible, pues la lectura que interesa es la puramente acusativa).

(39) María cogió (*de) los libros.

El español, sin embargo, presenta contraejemplos claros a (39), puesto que muchos objetos están precedidos por una preposición dativa, dependiendo de factores como la animacidad ${ }^{24}$.

(40) a. Compraron (a) una testigo.

b. Compraron $(* a)$ una casa.

Nótese que hay una diferencia fundamental entra la preposición dativa de los objetos directos (como los de 40) y la de los objetos indirectos: solo la segunda hace una aportación semántica (de tipo direccional).

(41) a. María regaló un libro a Juan.

b. María envió una carta a Juan.

El problema que los datos de (40) plantean para la propuesta de Pesetsky y Torrego 2004 no es tal si seguimos a Torrego 1998 al analizar la preposición $a$ de acusativo no como una verdadera preposición, sino

\footnotetext{
${ }^{23} \mathrm{La}$ presencia de esa segunda $\mathrm{T}$ entre $v^{*}$ y V se relaciona con las propuestas de Torrego 1995, 1998 sobre el doblado de clíticos.

${ }^{24}$ Simplifico mucho la cuestión, por motivos de espacio. Cf. Torrego 1998 para un estudio detallado de los factores que regulan la aparición de la $a$ de los objetos en español.
} 
como una marca de Caso. En términos sintácticos, eso es tanto como decir que la preposición a de los objetos directos no proyecta un sP, aunque, curiosamente, sí bloquea relaciones de concordancia; este último punto puede verse al comprobar los patrones de las pasivas reflejas y las pasivas impersonales. Solo en las primeras el verbo concuerda con el objeto:
(42) a. Se limpiaron las mesas.
Pasiva refleja
b. *Se limpiaron a los niños.
Pasiva impersonal

Debe notarse, en primer lugar, que las dos estructuras de (42) tienen un origen común: en ambas el clítico se elimina la aparición del agente (comoquiera que esto ocurra) y, por lo general, la asignación de acusativo. Sin embargo, cuando el verbo selecciona objetos preposicionales, como en (42b), la concordancia canónica (la que se espera por defecto) entre $\mathrm{T}$ y el SD objeto es imposible.

Torrego 1998 establece una diferencia adicional entre los objetos directos en relación con la preposición: $a$ puede ser una marca de Caso estructural o de Caso inherente. Basándose en observaciones procedentes de la gramática clásica, Torrego 1998 arguye que los objetos «afectados» (como el de 43b) reciben Caso acusativo inherente:

(43) a. María vio a Juan.

b. María acusó a Juan.

Torrego 1998, en particular, sugiere que verbos como acusarasignan Caso acusativo inherente a su objeto. Dentro de un marco como el de Hale y Keyser 2002 esto puede traducirse de la siguiente manera: los objetos que reciben acusativo inherente se generan en una configuración más compleja, similar a la de los verbos de locatum:

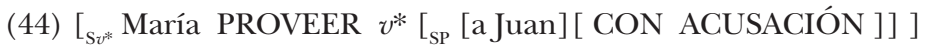

Los objetos que reciben acusativo estructural pertenecerían a estructuras más simples, como la de (45):

(45) $\left[\mathrm{S}_{v^{*}}\right.$ María $v^{*}$ [ ver [a Juan] ] ]

El análisis de Torrego 1998 tiene repercusiones en términos de (sub-) extracción: de ser correcta su propuesta, debería ser peor extraer desde un objeto con acusativo inherente que de un objeto con acusativo estructural, porque los primeros serían parecidos a los dativos y a los adjuntos, en el sentido de que recibirían Caso inherente. Los datos de (46), tomados de Torrego 1998, p. 37, demuestran que la predicción es correcta:

(46) a. ?El chico $\left.\left[{ }_{S C} \text { del que } e_{i} \text { han visto [a varias hermanas } t_{i}\right]_{]}\right]$

b. *El chico $\left[_{\mathrm{SC}}\right.$ del que ${ }_{\mathrm{i}}$ han acusado [a una hermana $\mathrm{t}_{\mathrm{i}}$ ] ]

El contraste de (46) nos dice que la preposición a no siempre ge- 
nera efectos de isla, lo cual, a su vez, nos obliga a relajar el contraste de (42) en términos de concordancia. Torrego 1995, p. 410, de hecho, demuestra que incluso los objetos preposicionales pueden concordar con los participios en determinadas estructuras:

(47) Tienen a los soldados castigados.

El dato de (45) demuestra que los objetos precedidos de $a$ no pueden concordar con $\mathrm{T}$ (como vimos en 42), pero pueden hacerlo con el participio castigados. Lo que en todo caso es imposible, como (48) hace evidente, es la concordancia con los objetos indirectos: la preposición dativa, al ser una marca de Caso inherente, haría de «escudo» para los rasgos- $\varphi$ del SD mis hijas.

(48) *Tengo dadas a mis hijas muchos libros.

De la discusión precedente puede concluirse (49).

(49) Condiciones sobre el Caso:

a. El Caso es un rasgo [ T] en D.

b. El Caso es estructural cuando D establece Acuerdo con T.

c. El Caso es inherente cuando D establece Acuerdo con P.

d. Los núcleos $\mathrm{T}\left(\mathrm{T}_{\text {SUETO }}\right.$ y $\mathrm{T}_{\text {OвIETO }}$ ) tienen una semántica de tipo «eventivo/temporal» (cf. Demirdache y Uribe-Etxebarria 2000).

e. Los núcleos P tienen una semántica de tipo léxico (en el sentido del análisis de las preposiciones de Hale y Keyser 2002).

La lista de (49) es una serie de observaciones sobre la Teoría del Caso, nada más. La más importante de estas observaciones es la que atañe al hecho de que $\mathrm{T}$ asigne Caso estructural y p Caso inherente. Si eso es así, lo que esperaríamos es que T y P difiriesen en su semántica -la de T sería (por así decirlo) más abstracta ${ }^{25}$. De hecho, la observación (49e) predice la inexistencia de preposiciones sin semántica propia (poniendo a un lado algunas versiones de $a$ y $d e$, que simplemente serían marcas de Caso -es decir-, la manera en que «resuena» la propiedad T que $\mathrm{D}$ posee).

La diferencia entre $\mathrm{T}$ y $\mathrm{P}$ tiene, como he dicho, repercusiones para procesos de (sub-) extracción; supongamos que formalizamos esa intuición de la siguiente manera:

(50) Condición de Extracción:

Solo un dominio que tenga un rasgo no interpretable $\mathrm{T}$ es transparente para operaciones de (sub-)extracción.

\footnotetext{
${ }^{25}$ Es muy probable que, en última instancia, la semántica de T y $\mathrm{P}$ sea la misma. En concreto, es probable que $\mathrm{T}$ y $\mathrm{P}$ puedan clasificarse como elementos de «coincidencia central» $\mathrm{y}$ «coincidencia terminal», en el sentido de Hale 1986. Así, por ejemplo, en (i) y (ii) tendríamos el mismo tipo de $\mathrm{T} / \mathrm{P}$, indicando coincidencia central (suponiendo que la información temporal de «presente» equivalga a «coincidencia central»):
}

(i) María baila.

(ii) María en Barcelona. 
(50) es suficiente para predecir que solo dos dominios pueden someterse a operaciones de (sub-) extracción: C y D. Además, (50) es consistente con el hecho de que los dominios encabezados por una $\mathrm{T}$ interpretable sean islas.

Nótese, no obstante, que (50) hace una predicción aún más interesante: si sp es una isla, sT también debería serlo. Esta idea es contraria a la idea de que solo sc y $\mathrm{S}^{*}$ (las fases) son islas, pero tiene cierto sentido en el contexto del sistema de Barreras de Chomsky 1986a; lo que quiero destacar aquí del trabajo de Chomsky 1986b, p. 15 es el estatus defectivo de st, que pese a ser lo que Chomsky entonces llamaba «Categoría de Bloqueo», no era una barrera en sí misma, sino por herencia de Sv.

Si sT/P es una barrera, la única manera de que esos dominios sean transparentes es mover el XP que queramos (sub-) extraer a una proyección externa a ST/P. Esto no es posible en el caso de los SPs, porque no hay ninguna Sonda que desplace los SDs a la Arista de P, pero sí lo es en el caso de las dos proyecciones de $\mathrm{T}$ propuestas en Pesetsky y Torrego 2004, porque tanto $\mathrm{C}^{-} \mathrm{T}_{\text {SUJ }}$ como $\mathrm{v}^{*}-\mathrm{T}_{\mathrm{OBJ}}$ pueden desempeñar ese papel. Esto nos da una explicación congruente del movimiento cíclico: un sx tiene que desplazarse fuera de las proyecciones de ST para no quedar aislado. Si tal idea resulta correcta, tenemos un argumento adicional para reforzar la noción de Arista ${ }^{26}$.
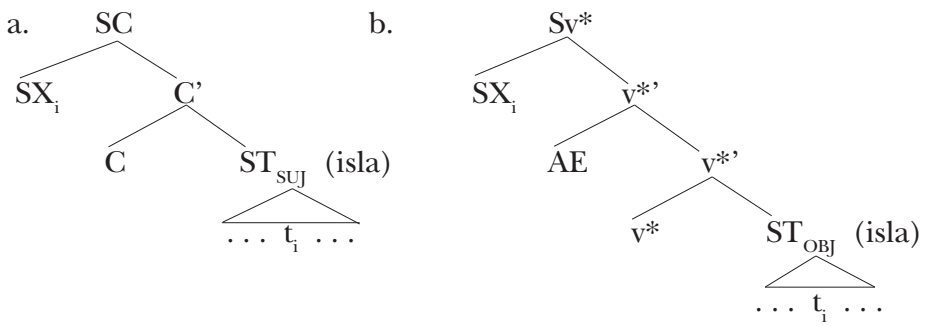

El análisis que acabo de esbozar resulta interesante por su capacidad unificadora (en términos de Caso) y tiene claras repercusiones para la posible relevancia del tiempo en la opacidad de algunas oraciones (piénsese, por ejemplo, en los efectos que puede tener que el verbo esté flexionado en indicativo, subjuntivo o infinitivo; cf. Chomsky 1986a). Hay muchos detalles en los que aquí no puedo entrar, pero me parece que los aspectos básicos de la propuesta han quedado bien establecidos; lo esencial, en mi opinión, es la idea de Pesetsky y Torrego 2001, 2004 de tratar a $\mathrm{T}$ y $\mathrm{P}$ como variantes de la misma megacategoría, en consonancia con trabajos pioneros como Emonds 1978 o Kayne 2000.

\footnotetext{
${ }^{26}$ Curiosamente, Chomsky 1986a, p. 11 comenta que sT no puede ser una isla, debido a la existencia de construcciones de MEc. Esto no es necesariamente un problema para la propuesta que defiendo aquí si, como demuestra Lasnik 1999, el SD que recibe Caso acusativo debe subir a la posición de objeto de la oración matriz.
} 


\section{Conclusiones}

En este artículo he intentado revisar la evolución de la Teoría del Caso a lo largo de los modelos teóricos de la gramática generativa, desde la noción de Rección de Chomsky 1981 hasta la de Acuerdo de Chomsky 2000, pasando por el efímero mecanismo de Atracción de Chomsky 1995. He concluido el trabajo haciendo referencia a la propuesta de Pesetsky y Torrego 2001 de que lo que llamamos Caso es en realidad un rasgo de Tiempo/Aspecto en D. Creo que esta propuesta tiene ventajas tanto conceptuales como empíricas sobre la de Chomsky: no solo defiende la idea de que todo rasgo formal tiene una interpretación potencial, sino que permite recuperar la noción de Caso inherente de otros modelos mediante la importancia que se confiere a la relación entre P y T. La teoría predice adecuadamente que un sp no puede ser un dependiente directo del verbo, debiendo analizarse como un segundo objeto (o un adjunto). Nótese, en este sentido, que estructuras como las de (52), tomadas de Bosque 1997, p. 140, no son un problema: Pesetsky y Torrego 2004 proponen que el Caso de D puede ser eliminado por una P, pero no prohíben que haya más elementos de este tipo; de hecho, podrá haber más de uno, siempre que los que sean adicionales hagan una contribución semántica.

(52) a. Quitó los libros [de [la cama]]

b. Quitó los libros [de [sobre la cama]]

c. Quitó los libros [de [encima de la cama]]

Pero hay más: si estoy en lo cierto, el hecho de que el Caso sea un rasgo $\mathrm{T}$ tiene consecuencias para los procesos de desplazamiento en general, puesto que T/P generaría zonas opacas para la extracción (islas), algo que nos proporcionaría una motivación adicional para la existencia de lo que Chomsky llama Arista.

Queda por investigar, claro está, si las dos propuestas (la de Chomsky y la de Pesetsky y Torrego) son unificables. Intuitivamente creo que sí, aunque los detalles me son difíciles de formular con precisión. La idea es bastante sugerente, si bien se piensa: tanto la concordancia como las preposiciones son elementos funcionales que «unen» dos objetos sintácticos. Considérese (53) a este respecto: tanto las marcas -os de (53a) como la preposición de de (53b) sirven para unir los sustantivos ministros y Argentina.

(53) a. Ministros argentinos.

b. Ministros de Argentina.

Prueba de que estas informaciones desempeñan un papel análogo es (54), que indica que la preposición de bloquea en efecto la concordancia: 
(54) Ministros (*de) argentinos.

Aunque en este momento no puedo ofrecer una formalización explícita, el paralelismo que he esbozado en (53) sugiere concebir las preposiciones como una especie de concordancia. Desde luego, las preposiciones tendrían más rasgos de los que tiene la concordancia (en particular, un rasgo interpretable T), pero la idea básica puede encontrarse, de hecho, en Torrego 1995, 1998: las preposiciones y los clíticos son una especie de «flexión».

La propuesta tiene sentido también en términos diacrónicos, pues, como es sabido, el desarrollo de las preposiciones en las lenguas románicas fue paralelo a la desaparición de las marcas flexivas. Dentro de la gramática generativa, esta idea tampoco es nueva: Kayne 1994, por ejemplo, analiza las preposiciones como elementos híbridos entre preposición-determinante-conjunción (cf. 55a), mientras que Uriagereka 2002 analiza algunas preposiciones como núcleos proyecciones de concordancia en estructuras posesivas (cf. 55b):

(55) a. $\left[{ }_{\mathrm{SD}} \mathrm{La}\left[\left[_{\mathrm{SP} / \mathrm{D}}\right.\right.\right.$ voiture $_{\mathrm{i}}\left[\left[_{\mathrm{P} / \mathrm{D}}\right.\right.$, de $\left[{ }_{\mathrm{ST}}\right.$ Jean $\left.\left.\left.\left.\mathrm{t}_{\mathrm{i}}\right]\right]\right]\right]$

b. $\left[_{\mathrm{SD}}\right.$ The $\left[{ }_{\mathrm{SConc}}\right.$ poor neighborhoods $\left[_{\mathrm{C}}\left[_{\text {Conc }}\right.\right.$ of $[$ the city $\left.\left.\left.\left.] \mathrm{t}_{\mathrm{i}}\right]\right]\right]\right]$

Dejo aquí esta sugerencia, esperando desarrollarla en el futuro. De ser posible una unificación de las dos teorías, no solo estaríamos ante un nuevo paso adelante en el objetivo particular de la lingüística generativa (explicar por qué la facultad del lenguaje es como es, y en nuestro caso, por qué manifiesta propiedades tan peculiares como la morfología), sino ante un nuevo paso adelante en el objetivo de toda disciplina científica: demostrar que fenómenos aparentemente inconexos tienen una explicación común.

\section{REFERENCIAS BIBLIOGRÁFICAS}

Belletti, A. (1988): «The Case of unaccusatives», Linguistic Inquiry 19, pp. 1-34. - (1990): Generalized verb movement, Turín, Rosenberg and Sellier.

Boeckx, C. (1999): «Conflicting C-command requirements», Studia Linguistica 53, pp. $227-250$.

— (2000): «Quirky agreement», Studia Linguistica 54, pp. 354-380.

- (2003): Islands and chains, Amsterdam, John Benjamins.

BosQue, I. (1997): «Preposición tras preposición», en Almeida, M. y Dorta, J. (eds.), Contribuciones al estudio de la lingüistica hispánica. Homenaje al profesor Ramón Trujillo, vol. 1, Tenerife, Montesinos, pp. 133-155.

Сномsкy, N. (1955/1975): The logical structure of linguistic theory, Nueva York, Plenum.

- (1973): «Conditions on transformations», en Anderson, S. y Kiparsky, P. (eds.), A Festschrift for Morris Halle, Nueva York, Holt, Renehart and Winston, pp. 232-286. 
- (1981): Lectures on government and binding, Dordrecht, Foris.

- (1982): Some concepts and consequences of the theory of government and binding, Cambridge (Mass.), Miт Press.

- (1986a): Barriers, Cambridge (Mass.), мiт Press.

- (1986b): Knowledge of language. Its nature, origin, and use, Londres, Praeger.

- (1991): «Some notes on economy of derivation and representation», en Friedin, R. (ed.), Principles and parameters in comparative grammar, Cambridge (Mass.), Miт Press.

— (1993): «A minimalist program for linguistic theory», en Hale, K. y Keyser, S. (eds.), The view from building 20: essays in linguistics in honor of Sylvain Bromberger, Cambridge (Mass.), мIт Press, pp. 1-52.

- (1995): The minimalist program, Cambridge (Mass.), мIт Press.

- (2000): «Minimalist inquiries: the framework», en Martin, R. y otros (eds.), Step by step. Essays on minimalist syntax in honor of Howard Lasnik, Cambridge (Mass.), MIT Press, pp. 89-155.

- (2001): «Derivation by phase», en Kenstowicz, M. (ed.), Ken Hale: A life in language, Cambridge (Mass.), Miт Press, pp. 1-52.

- (2004): «Beyond explanatory adequacy», en Belletti, A. (ed.), Structures and beyond. The cartography of syntactic structures (vol. 3), Oxford (Nueva York), Oxford University Press, pp. 104-131.

- (2008): «On phases», en Freidin, R. y otros (ed.), Foundational issues in linguistic theory: essays in honor of Jean-Roger Vergnaud, Cambridge (Mass.), MIT Press.

—y LASNik, H. (1977): «Filters and control», Linguistic Inquiry 8, pp. 425-504.

- (1995): «The theory of principles and parameters», en Chomsky, N., The minimalist program, Cambridge (Mass.), MIT Press, pp. 13-127.

Demirdache, H. y Uribe-Etxebarria, M. (2000): «The primitives of temporal relations», en Martin, R. y otros (eds.), Step by step. Essays on minimalist syntax in honor of Howard Lasnik, Cambridge (Mass.), MIT Press, pp. 157-186.

Emonds, J. (1985): A unified theory of syntactic categories, Foris, Dordrecht.

Frampton, J., S. Gutmann, J. Legate y Yang, C. (2000): "Remarks on derivation by phase: feature valuation, agreement, and intervention», manuscrito inédito, MIT.

Gallego, A. (2007): Phase theory and parametric variation, tesis doctoral inédita, UAB.

Gallego, A. y Uriagereka, J. (2007): «Conditions on sub-extraction», en Eguren, L. y Fernández-Soriano, O. (eds.), Coreference, modality, and focus. Studies on the syntax-semantics interface, Amsterdam, John Benjamins, pp. 45-70.

Hale, K. (19860): «Notes on world view and semantic categories: some Warlpiri examples», en Muysken, P. y van Riemsdijk, H. (eds.), Features and projections, Dordrecht, Foris, pp. 233-254.

- y Keyser, S. (2002): Prolegomenon to a theory of argument structure, Cambridge (Mass.), Mit Press.

Hiraiwa, K. (2005): Dimensions of symmetry in syntax: agreement and clausal architecture, tesis doctoral inédita, MIT.

Hornstein, N. (2003): «On control», en Hendrick, R. (ed.), Minimalist syntax, Oxford, Blackwell, pp. 6-81.

KAYNE, R. (1989): «Facets of past participle agreement in Romance», en Benin- 
cà, P. (ed.), Dialect variation and the theory of grammar, Dordrecht, Foris, pp. 85-104.

- (1994): The antisymmetry of syntax, Cambridge (Mass.), MIT Press.

- (2000): Parameters and universals, Nueva York, Oxford University Press.

Korzumi, M. (1995): Phrase structure in minimalist syntax, tesis doctoral inédita, MIT.

LASNIK, H. (1999): Minimalist analysis, Oxford, Blackwell.

LegATE, J. (2003): «Some interface properties of the phase», Linguistic Inquiry 34 , pp. 506-516.

Masullo, P. (1992): «Quirky datives in Spanish and the non-nominative subject parameter», en Kathol, A. y Beckman, J. (eds.), MIT working papers in linguistics 16, pp. 89-103.

Pesetsky, D. y Torrego, E. (2001): «T-to-C movement: causes and consequences», en Kenstowicz, M. (ed.), Ken Hale: a life in language, Cambridge (Mass.), MIT Press, pp. 355-426.

- (2004): «Tense, case, and the nature of syntactic categories», en Guéron, J. y Lecarme, J. (eds.), The syntax of time, Cambridge (Mass.), MIT Press, pp. 495-537.

— (2007): «The syntax of valuation and the interpretability of features», en Karimi, S. y otros (eds.), Phrasal and clausal architecture. Syntactic derivation and interpretation, Amsterdam, John Benjamins, pp. 262-294.

Pollock, J. I. (1989): «Verb movement, universal grammar, and the structure of the IP», Linguistic Inquiry 20, pp. 365-424.

Postal, P. (1974): On raising: one rule of english grammar and its theoretical implications, Cambridge (Mass.), MIT Press.

Rivero, M. L. (2004): «Spanish quirky subjects, person restrictions, and the person-case constraint», Linguistic Inquiry 35, pp. 494-502.

Rizzi, L. (1990): Relativized minimality, Cambridge (Mass.), miт Press.

- (1997: «The fine structure of the left periphery», en Haegeman, L. (ed.), Elements of grammar. Handbook in generative syntax, Dordrecht, Kluwer, pp. 281-337.

Svenonius, P. (2002): «Case is uninterpretable aspect», Proceedings of perspective on aspect conference, Utrecht, University of Utrecht.

Torrego, E. (1995): «On the nature of cliting doubling», en Campos, H. y Kempchinsky, P. (eds.), Evolution and revolution in linguistic theory: studies in honor of Carlos P. Otero, Washington, Georgetown University Press, pp. 399-418.

— (1996): «Experiencers and raising verbs», en Friedin, R. (ed.), Current issues in comparative grammar, Dordrecht, Kluwer, pp. 101-120.

- (1998): The dependencies of objects, Cambridge (Mass.), MIт Press.

- (1999): «Aspect in the prepositional system of romance», en Satterfield, T. y otros (eds.), Current issues in Romance languages, Amsterdam, John Benjamins, pp. 337-357.

URIAGEREKA, J. (1988): On government, tesis doctoral inédita, Univ. Connecticut.

- (1998): Rhyme and reason, Cambridge (Mass.), MIT Press.

- (2002): «From being to having», en Uriagereka, J., Derivations. Exploring the dynamics of syntax, Londres/Nueva York, Routledge, pp. 192-211. 